\title{
Tratamiento endocrinológico de la endometriosis
}

\author{
Ariel Iván Ruiz Parra*
}

RESUMEN: Las modalidades actualmente disponibles para ẹ tratamiento de la endometriosis son: la videolaparoscopia operatoria, la microcirugía, la cirugía, el tratamiento médico endocrinológico, la fertilización asistida y la analgesia con seguimiento estrecho. Aquí se discuten los fundamentos del tratamiento endocrinológico, la forma de administración y sus ventajas y desventajas, para orientar al médico en la elección individualizada de una terapia.

PALABRAS CLAVES: Endometriosis, tratamiento médico, progestágenos, danazol, análogos de la GnRH.

SUMMARY: The treatment modalities for endometriosis include: operative laparoscopy, microsurgery, surgery, pharmacologyc treatment, assisted reproduction techniques, and analgesia with close observation. In this article, the rationale for endocrine therapy, the forms of administration, and the advantages and disadvantages are discussed, in order to guide the physician into the individual choice of a treatment.

KEY WORDS: Endometriosis, medical therapy, progestogens, danazol, GnRH agonists.

\section{Introducción}

La endometriosis es la presencia de tejido endometrial en una localización ectópica. Con frecuencia se considera que se requiere tanto la presencia de glándulas como de tejido estromal para establecer el diagnóstico de la enfermedad desde el punto de vista histológico (1). Las lesiones de endometriosis se encuentran mas frecuentemente sobre los ligamentos uterosacros, ovarios, peritoneo pélvico, intestino y apéndice (2). La entidad se asocia frecuentemente con dismenorrea, dispareunia, dolor pélvico crónico e infertilidad. La prevalencia de la endometriosis en la población general es difícil de determinar. Esta varía entre el 7 y el $50 \%$ de acuerdo con la indicación con la cual se realicen procedimientos tales como la laparoscopia y la laparotomía y de la experiencia del cirujano para reconocer la enfermedad (3). Sin embargo, se estima que en promedio la endometriosis afecta a una de cada 10 a 15 mujeres en edad reproductiva $(1,2)$.

\section{Bases del tratamiento endocrinológico de la endometriosis}

Durante más de 50 años se ha utilizado el tratamiento quirúrgico conservativo en el tratamiento de la endometriosis. En la actualidad la cirugía está indicada cuando se encuentra un endometrioma y en pacientes con infertilidad y endometriosis en estados III y IV (4). Sin embargo,

* Ginecólogo-endocrinólogo.

Profesor asociado, Facultad de Medicina.

Universidad Nacional de Colombia. el riesgo de desarrollar adherencias posoperatorias es alto, siendo el ovario especialmente vulnerable al trauma quirúrgico $(5,6)$. Si el tratamiento quirúrgico falla en lograr el embarazo, la fertilización in vitro puede ser exitosa (4). En el momento el tratamiento inicial de elección para la endometriosis (hasta estado III) consiste en practicar intervenciones por vía laparoscópica en el momento del diagnóstico y plantear porteriormente tratamientos endocrinológicos complementarios. Para las mujeres con endometriosis y dolor pélvico, el tratamiento quirúrgico produce solamente un alivio temporal y hay una alta tasa de recurrencia del dolor. Por otro lado, el tratamiento hormonal de la endometriosis produce alivio del dolor en la mayoría de las pacientes; así, el control del dolor constituye actualmente la indicación primaria de la terapia hormonal de la endometriosis $(2,4)$.

El tratamiento endocrinológico de la endometriosis se fundamenta en el hecho de que el crecimiento, mantenimiento, función y diseminación de la endometriosis se estimulan con la secreción cíclica de las hormonas esteroides ováricas $(4,7,8)$. Como en el endometrio eutópico, también existen receptores de estrógenos y de progesterona en el tejido endometriósico, aunque su concentración es un 50 a $70 \%$ menor que en el endometrio normal y no presentan variaciones durante el ciclo menstrual $(4,9,10)$. También existen receptores para los andrógenos en el tejido endometriósico $(4,10)$. Por lo tanto, los efectos benéficos de los tratamientos con esteroides pueden ser consecuencia de acciones hormonales directas mediadas a través de receptores hormonales, consecuencias indirectas de los cambios en las concentraciones circulantes de esteroides o una combinación de ambas acciones (10). Por otro lado, en el tejido 
endometrial también se han encontrado factores de crecimiento tales como el factor de crecimiento epidérmico, factor transformante del crecimiento, factor estimulante de colonias y factores de crecimiento fibroblástico y éstas proteínas podrían ser reguladores importantes del crecimiento y la diferenciación endometrial (4). El estradiol y el factor de crecimiento epidérmico pueden actuar sinérgicamente para estimular la proliferación del endometrio humano (4).

\section{Tratamiento con anticonceptivos orales}

El término "seudoembarazo", introducido por Kistner en 1958, indica un estado de amenorrea hiperhormonal inducida por la administración continua de preparados de estrógenos y progestágenos potentes (11). El efecto benéfico de los preparados combinados de estrógenos y progestágenos en el tratamiento de la endometriosis depende de su administración continua, de las propiedades predominantemente progestacionales del compuesto y del estado de amenorrea hiperhormonal que inducen (11). El tratamiento tiene los siguientes efectos (11): a) Supresión de la secreción hipotalámica de GnRH y de la secreción hipofisiaria de gonadotropinas; b) Como consecuencia, supresión de la foliculogénesis y la esteroidogénesis ovárica; c) El endometrio y el tejido endometriósico sufren una estimulación inicial, hipertrofia, aumento de la vascularidad y edema, los progestágenos provocan cambios deciduales y amenorrea; mas tardíamente ocurre necrobiosis y reabsorción del tejido endometriósico.

\section{A. Dosis y administración}

Los anticonceptivos de elección son aquellos que tienen propiedades fuertemente progestacionales. Es preferible comenzar con la dosis más baja (30 a $35 \mathrm{~g}$ de etinilestradiol al día) y aumentarla a dos o mas tabletas diarias en forma progresiva si ocurre sangrado transhormonal. La dosis de mantenimiento es la dosis mas baja que produzca amenorrea (12). Los anticonceptivos se utilizan en forma continua durante 8 a 9 meses o más dependiendo del estado inicial de la enfermedad y de la respuesta at tratamiento. Al finalizar el tratamiento la ovulación y la menstruación reaparecen en 4 a 6 semanas $(12,13)$.

\section{B. Efectos colaterales}

Los efectos colaterales ocurren hasta en un $87 \%$ de las pacientes, la tolerancia es mala y la tasa de interrupción de la terapia es mayor del 30\% (11). Durante los dos o tres meses iniciales la mayoría de las pacientes refieren aumento de los síntomas de la enfermedad, además de las molestias relacionadas especificamente con la administración de las hormonas (12). Los efectos colaterales incluyen: náuseas, distensión abdominal, depresión, dolor y sensibilidad en los senos, edema y aumento de peso, sangrado transhormonal y aparición de várices superficiales (12). Por éstas razones y por la baja eficacia, en la actualidad el tratamiento con anticonceptivos orales se usa rara vez y estaría indicado solo en pacientes seleccionadas en las cuales hay contraindicaciones o no se pueden disponer de medicamentos alternos.

\section{Resultados}

La mayoría de los informes sobre tratamiento de la endometriosis con anticonceptivos orales son estudios clínicos subjetivos y no controlados. La respuesta sintomática ha variado desde un "porcentaje bajo" hasta un 93\% y la tasa corregida de embarazos va desde un 10.5 hasta un $54 \%$ $(11,13)$. Sin embargo, cuando se comparó un anticonceptivo oral a base de mestranol y noretinodrel con el danazol, el alivio sintomático fue de solo un $30 \%$ con el anticonceptivo comparado con un $86 \%$ con el danazol (13).

\section{Tratamiento con progestágenos}

Los progestágenos solos se han utilizado por mas de dos décadas para el tratamiento de la endometriosis. El mecanismo de acción de éstos agentes incluye la inducción de un medio ambiente hipoestrogénico acíclico por supresión de las gonadotropinas, inhibición de la ovulación e inducción de amenorrea (12). Los progestágenos pueden actuar solamente en células endometriales que posean receptores de progesterona inducidos por los estrógenos. En éstas células los progestágenos inducen cambios secretores seguidos de transformación decidual con la necrosis subsecuente $(11,14)$. Las principales ventajas de los progestágenos solos sobre los anticonceptivos orales son la mejor tolerancia del tratamiento y la ausencia de las complicaciones propias de la terapia estrogénica. Los progestágenos están indicados principalmente en la endometriosis leve y moderada.

\section{A. Acetado de medroxiprogesterona}

1. Dosis y administración. La terapia progestacional mas comúnmente usada para el tratamiento de la endometriosis es la administración de acetato de medroxiprogestrona (AMP). El AMP puede utilizarse en forma inyectable de depósito a dosis de $100 \mathrm{mg}$ cada dos semanas por 4 dosis, seguidas de $200 \mathrm{mg}$ cada mes durante 4 meses mas. También puede utilizarse por vía oral a dosis de 30 a $100 \mathrm{mg}$ al día en forma continua durante 3 a 6 meses $(14,15)$. Al parecer las dosis mas altas no disminuyen la incidencia de sangrado transhormonal ni aumentan la eficacia (14); por ésta razón, nuestro esquema consiste en administrar dosis iniciales de $20 \mathrm{mg} /$ día de AMP por vía oral y aumentar la dosis progresivamente hasta lograr la amenorrea; la mayoría de nuestras pacientes reciben entre 30 y $40 \mathrm{mg} /$ día de AMP por vía oral. La administración del AMP de depósito por vía parenteral se asocia con anovulación y amenorrea prolongadas al terminar el tratamiento por lo que no se considera de elección en mujeres que desean fertilidad. Por el contrario, la administración del medicamento por vía oral no conlleva anovulación prolongada al terminar el tratamiento.

2. Efectos colaterales. Los efectos colaterales mas importantes son: sangrado genital de tipo manchado en aproximadamente el $50 \%$ de las pacientes, depresión en un 5 a 10\%, sensibilidad mamaria, retención de líquidos, 
aumento de peso, distensión abdominal y náuseas $(11,12,14)$. Las dosis altas de AMP suprimen la SHBG y aumentan la testosterona libre pero en menor proporción que el danazol; igualmente, pueden presentarse modificaciones de las lipoproteínas consistentes principalmente en disminución de las HDL (14).

3. Resultados. Se ha informado una disminución del $68 \%$ en los puntajes de la clasificación de la American Fertility Society (AFS) al final de la terapia y mejoría del dolor pélvico en un $80 \%$ de las pacientes (16). También se ha demostrado que la terapia con AMP redúce la inflamación intraperitoneal asociada con la endometriosis (13). En estudios comparativos, el AMP ha resultado ser tan efectivo como el danazol para el tratamiento de la endometriosis (12). En un estudio prospectivo, doble ciego y controlado con placebo para comparar la eficacia del danazol ( $600 \mathrm{mg} / \mathrm{día} / 6 \mathrm{meses})$ con la eficacia de dosis altas de AMP (100 mg/día/6 meses), se encontró que ambos medicamentos aliviaban significativamente el dolor y disminuían el puntaje de endometriosis comparados con el placebo y que no hubo diferencias significativas entre el danazol y el AMP en ningún parámetro evaluado en cualquier momento (10). Los efectos benéficos sobre la dismenorrea y otros síntomas secundarios a la endometriosis fueron duraderos: 6 meses después del tratamiento con danazol o AMP los síntomas fueron significativamente mas leves en su intensidad que en las mujeres tratadas con placebo (10). En cuanto a las tasas de fertilidad, tampoco se han encontrado diferencias significativas en el tratamiento de la endometriosis entre AMP, danazol y placebo y se puede concluir que ninguno de los tratamientos médicos aumenta significativamente la fertilidad de las mujeres con endometriosis $(10,12,15)$. Por éstas razones en pacientes con limitaciones económicas nosotros consideramos al AMP por vía oral o parenteral como primera elección para el tratamiento de la endometriosis leve a moderada.

\section{B. Gestrinona}

La gestrinona es un derivado de la 19-nortestosterona designado como 13ß-etil-17ß-hidroxi-18,19 dinorpreganano-4,9,11 trien-ona (17). El compuesto y sus metabolitos hidroxilados activos tienen actividad antiestrogénica, antiprogestacional y actividad androgénica (17). La gestrinona inhibe la foliculogénesis y suprime el aumento de las gonadotropinas a mitad del ciclo menstrual $\mathrm{y}$, en consecuencia, produce disminución de los estrógenos, anovulación y amenorrea. Es posible que la gestrinona actúe a través de ésta disminución del soporte estrogénico del tejido endometriósico o que tenga efectos directos debidos a su actividad antiestrogénica, antiprogestacional y androgénica (18). La acción antiestrogénica se debe a que la gestrinona disminuye la concentración de receptores de estrógenos y su reciclaje a nivel endometrial; éste efecto antiestrogénico puede ser el responsable de la atrofia endometrial a pesar de la presencia de concentraciones de estradiol propias de la fase folicular (19). Por otro lado, la gestrinona tiene actividad androgénica intrínseca, parcialmente debido a un aumento en la proporción de la testosterona libre por disminución de la globulina transportadora de esteroides sexuales (SHBG) o por competencia con sus sitios de unión. Este efecto probablemente es menor ya que menos del $10 \%$ de la testosterona unida a la SHBG puede ser desplazada por la gestrinona a las dosis terapéuticas (19).

1. Dosis y administración. La dosis por vía oral va de $2.5 \mathrm{mg}$ dos a tres veces por semana a $5 \mathrm{mg}$ dos veces por semana durante 6 meses (17). El tratamiento debe iniciarse el primer día o dentro de los tres primeros días de la menstruación (19). Después de terminar el tratamiento, el retorno de la menstruación ocurre entre 15 y 62 días. La forma de administración de éste medicamento dos a tres veces por semana mejora el cumplimiento de la paciente. También puede administrarse por vía vaginal a las dosis de 2.5 a $5 \mathrm{mg}$ cada dos días. La administración vaginal es tan efectiva como la oral y causa menos efectos colaterales, por lo que puede utilizarse cuando la paciente tiene gastritis, enfermedad ulceropéptica, hiperacidez o intolerancia a los esteroides. También se benefician de la administración de gestrinona por vía vaginal las pacientes que desarrollan efectos androgénicos (17). Además se han desarrollado cápsulas subdérmicas de silastic que liberan lentamente la gestrinona durante un período de un año. Los implantes miden $3 \mathrm{~cm}$ y contienen 30 o $40 \mathrm{mg}$ de gestrinona. A cada paciente se le insertan 8 implantes a través de un trocar utilizando anestesia local. La tasa de fertilidad es comparable con la de las otras vías de administración y los efectos colaterales son menos intensos en las mujeres que utilizan el implante (17).

2. Efectos colaterales. Se asocian con la actividad moderadamente androgénica e incluyen: seborrea (70 a $80 \%$ ), acné ( 65 a $80 \%)$, hirsutismo (10 a $15 \%$ ), atrofia de los senos (30\%), edema (25\%), engrosamiento de la voz (menos del 10\%), aumento de peso probablemente por aumento de la masa muscular y del tejido adiposo, edema articular, caída del cabello, aumento de la líbido, sangrado genital, cefalea y palpitaciones $(4,14,17-19)$. La gestrinona, como el danazol disminuyen las HDL y aumentan las LDL; la gestrinona además disminuye los triglicéridos y las VLDL. Esta disminución de las VLDL puede ser un efecto antiestrogénico de la gestrinona que causa inhibición de su síntesis hepática. Las modificaciones en las lipoproteínas son en total aterogénicas y ameritan vigilancia estricta (19).

3. Resultados. Se ha informado una tasa de alivio sintomático del 85 al $90 \%$ y una tasa de embarazos del $59 \%(17)$. La reducción del puntaje de endometriosis es de alrededor de un $47 \%$ y la mayoría de las pacientes experimentan alivio de los síntomas durante los primeros dos meses (19). En un estudio comparativo entre gestrinona y placebo se encontraron diferencias estadísticamente significativas tanto en las tasas de mejoría ( $83 \%$ con gestrinona vs $29 \%$ con placebo), como en las tasas de progresión de la enfermedad (0 en el grupo tratado vs $47 \%$ en el grupo placebo) (20). En otro estudio comparativo entre placebo y gestrinona se observó que en las mujeres no tratadas la enfermedad se mejoró o desapareció en aproximadamente la mitad de los casos, pero ésta tasa fue significativamente mayor en el grupo tratado con gestrinona (21). También se encontró progreso de la 
enfermedad únicamente en algunas pacientes del grupo placebo, mientras que no ocurrió aumento de la enfermedad en ninguna paciente del grupo tratado (21). En un estudio comparativo entre danazol, gestrinona, linestrenol y buserelin para el tratamiento preoperatorio de la endometriosis ovárica, la gestrinona y el danazol tuvieron efectos similares y el buserelin fue superior a los demás para inducir la regresión de las lesiones ováricas de la endometriosis (22).

Al parecer no hay diferencias en la tasa de embarazos al comparar las diversas dosis de gestrinona (17). En estudios comparativos con danazol tampoco se encontraron diferencias significativas en cuanto hace referencia a la tasa de embarazos (12). De igual forma, en un estudio comparativo entre gestrinona, danazol y buserelin o goserelin no se encontraron diferencias significativas en la tasa de embarazos en un seguimiento de 6 meses después del tratamiento (23).

\section{Tratamiento con danazol}

El danazol es el derivado 2-3 Isoxasol de la 17- etiniltestosterona. El medicamento tiene múltiples sitios y mecanismos de acción $(8,11,13,14,24)$ :

1. El danazol inhibe en forma reversible las enzimas 20-22 desmolasa, 3ß-hidroxiesteroide deshidrogenasa, 17 -hidroxilasa, 21-hidroxilasa, 11ß-hidroxilasa y 17cetoesteroide-oxidoreductasa de la vía sintética de los esteroides. El danazol puede ligarse directamente al sitio de unión del esteroide del sistema enzimático del citocromo P-450, estableciendo una inhibición de tipo competitivo. Las mujeres que reciben dosis terapéuticas de danazol alcanzan concentraciones circulantes que se encuentran en el rango requerido para inhibir todas éstas enzimas de la esteroidogénesis.

2. El danazol puede unirse directamente a receptores de andrógenos, progesterona y glucocorticoides pero no se une en forma importante a los receptores de estrógenos. Como consecuencia de su unión a los receptores de glucocorticoides, el danazol puede suprimir el sistema inmune de una manera similar a como lo hacen los glucocorticoides. El medicamento inhibe la proliferación de linfocitos en cultivos activados con mitógenos para las células $\mathrm{T}$.

3. El danazol se une a la SHBG y a la globulina transportadora de corticosteroides. Así, una parte de las propiedades androgénicas del medicamento se debe a un aumento de la testosterona libre del 1 al $2 \%$ por desplazamiento de la SHBG. Pero además, actuando directamente como un agonista sobre el receptor de andrógenos, el compuesto suprime el crecimiento del endometrio y del tejido endometriósico. Los efectos androgénicos directos e indirectos acoplados con la carencia de propiedades estrogénicas del danazol, inhiben el crecimiento endometrial y conducen a la atrofia de los implantes endometriósicos.

4. El danazol no altera la concentración de FSH, pero puede producir aumentos significativos de la LH en mujeres con ciclos menstruales normales. El medicamento disminuye la frecuencia de los pulsos de $\mathrm{LH}$, pero aumenta la amplitud de los mismos.
5. El danazol inhibe el desarrollo de un folículo dominante y anula la elevación de la LH y de la FSH que ocurre a mitad del ciclo con lo cual suprime también la ovulación.

Los diversos mecanismos de acción descritos para el danazol se integran en la siguiente forma: El danazol produce un medio ambiente altamente androgénico debido a que tiene propiedades androgénicas intrínsecas, disminuye la síntesis de SHBG y desplaza la testosterona de su unión con la SHBG. Este medio androgénico inhibe directamente el crecimiento de la endometriosis. El medicamento produce además un medio ambiente hipoestrogénico por inhibición del crecimiento folicular como consecuencia de acciones directas sobre el ovario y el eje hipotálamo-hipófisis. El ambiente endocrino acíclico producido por el danazol minimiza la posibilidad de menstruación y la siembra de nuevos implantes de endometriosis en el peritoneo (24).

\section{A. Dosis y administración}

El danazol se debe iniciar después de completar la menstruación con el fin de evitar su administración inadvertida durante la gestación ya que puede provocar virilización de fetos femeninos. La dosis de $200 \mathrm{mg} /$ día ofrece alivio del dolor, pero se requiere un mínimo de 400 $\mathrm{mg}$ /día para suprimir la ovulación y producir amenorrea (24). La dosis promedio se encuentra alrededor de 600 $\mathrm{mg}$ /día $200 \mathrm{mg}$ tres veces al día); sin embargo, las dosis se deben ajustar individualmente de acuerdo con la respuesta en términos de supresión de la menstruación; la dosis mínima efectiva es aquella que cause amenorrea a la paciente. Las mujeres con estados I y II de endometriosis responden a dosis de 200 a $400 \mathrm{mg} /$ día de danazol; las pacientes con estados III y IV requieren dosis de 600 a 800 $\mathrm{mg} /$ día (4). La duración promedio del tratamiento es de 6 meses, pero también debe ser individualizada: en pacientes en quienes se planea una cirugía conservadora, se obtienen los máximos beneficios con un curso previo de danazol de 3 meses; por el contrario, en las pacientes que están siendo tratadas por dolor pélvico la terapia se puede extender por 4 a 9 meses. La menstruación retorna a las 4 semanas después de suspender una dosis diaria de $400 \mathrm{mg}$ de danazol y a las 5 semanas después de $800 \mathrm{mg} /$ día (13).

\section{B. Efectos colaterales}

Ocuren hasta en un $85 \%$ de las pacientes (25); casi todos éstos efectos indeseables se deben a las propiedades androgénicas del danazol. Puede ocurrir: aumento de peso $(69 \%)$, calambres musculares $(52 \%)$, atrofia de los senos (48\%), bochornos (42\%), cambios de humor (38\%), seborrea $(37 \%)$, depresión (32\%), edema $(28 \%)$, acné $(27 \%)$, fatiga $(25 \%)$, hirsutismo $(21 \%)$, cefalea $(17 \%)$, rash $(8 \%)$, engrosamiento de la voz $(7 \%)$, distensión abdominal, y toxicidad hepática, incluyendo hepatitis colestásica e ictericia $(14,25)$. Los programas de ejercicios y dieta desde el inicio de la terapia pueden ayudar a prevenir algunos de éstos efectos colaterales; una reducción de las dosis del medicamento también puede contribuir a disminuir los efectos indeseables. 
Las propiedades androgénicas del danazol provocan una disminución significativa de las HDL y un aumento de las LDL; las concentraciones de colesterol regresan a lo normal en uno a cinco meses después de interrumpir el tratamiento (24). También se ha observado resistencia a la insulina en mujeres que reciben danazol. Las acciones del medicamento a nivel hepático provocan además un aumento de prealbúmina, inhibidor de $\mathrm{C}_{1}$-estearasa, haptoglobina, transferrina, antitrombina III, protrombina y plasminógeno y una disminución de la SHBG y de la TBG. En consecuencia, hay un aumento de la testosterona $\mathrm{y}$ de la $\mathrm{T}_{4}$ libres con el danazol (24).

El danazol está contraindicado en mujeres gestantes o lactantes; cuando hay una hemorragia genital cuya etiología no se conoce o cuando hay compromiso severo de la función hepática, renal o cardíaca. El danazol se debe evitar en pacientes adolescentes antes de que completen su crecimiento y desarrollo y debe evitarse también en pacientes con hipercolesterolemia severa o historia de enfermedad cardiovascular arterioesclerótica (14).

\section{Resultados}

Ocurre alivio del dolor en un $90 \%$ de las pacientes tratadas con danazol. Los puntajes (AFS) de la endometriosis disminuyen en un 50\% aproximadamente (24). La respuesta al danazol depende de la dosis. En un estudio que incluyó mas de 440 pacientes tratadas con buserelin y mas de 80 mujeres tratadas con danazol, se demostró que ambos tratamientos eran altamente eficaces para el tratamiento de la endometriosis (26). Sin embargo, los efectos colaterales fueron mejor tolerados por las pacientes tratadas con el buserelin (26). En otro estudio comparativo entre danazol, láser de $\mathrm{CO}_{2}$ y danazol + laser de $\mathrm{CO}_{2}$, para el tratamiento de la endometriosis estado I (AFS), no se encontraron diferencias estadísticamente significativas en cuanto hace referencia a las tasas de embarazos (27). En éste estudio la tasa promedio de embarazos de los tres tipos de tratamiento fue del $47.3 \%$ (27). En forma interesante, en un estudio en el cual se investigó la capacidad de concebir por segunda vez sin tratamiento, después de haber logrado un primer embarazo con un tratamiento previo para la endometriosis con danazol, laser por Laparoscopia, cirugía conservativa, cirugía + danazol o sin tratamiento, se encontró que la probabilidad de un segundo embarazo es muy alta $(76 \%)$ independientemente del método de tratamiento, la duración de la infertilidad previa y la edad de la paciente (28). En términos generales no hay evidencia de que el danazol sea superior al manejo expectante de la endometriosis en cuanto hace referencia a la tasa de embarazos (15). Como ocurre con otros tipos de tratamiento médico de la endometriosis, se presentan recurrencias de la enfermedad desde el primer año después de suspender la terapia.

En un estudio comparativo entre el danazol y un agonista de la GnRH, se encontró que únicamente el danazol reducía los niveles de autoanticuerpos en mujeres con endometriosis. Los autoanticuerpos que mostraron un descenso al final del tratamiento con danazol incluían entre otros: IgG anticardiolipina, IgG anti-fosfatidilserina, IgG, IgM e IgA antifosfatidiletanolamnina e IgG e IgM anti-fosfatidilinositol (29). Estos autores encontraron además que solamente las pacientes con concentraciones normales de autoanticuerpos desde el comienzo o en quienes el danazol normalizó la concentración de autoanticuerpos, lograron el embarazo. Una gestación que ocurrió en una paciente con concentraciones elevadas de autoanticuerpos terminó en aborto (29). Estas anormalidades inmunológicas pueden estar relacionadas causalmente con la infertilidad que se observa en las mujeres con endometriosis (30).

\section{Tratamiento con análogos agonistas de la GnRH}

\section{A. Metabolismo de la GnRH}

La hormona liberadora de gonadotropinas $(\mathrm{GnRH})$ es un decapéptido cuya estructura terciaria tiene aspecto de "V", con el vértice situado en la unión de los aminoácidos tirosina ${ }^{5}$-glicina ${ }^{6}(31)$. El receptor para la GnRH, localizado en la membrana del gonadotropo, reconoce el conjunto de la molécula, pero la afinidad depende especialmente de los aminoácidos piroglutámico ${ }^{1}$, glicina ${ }^{6}$ y glicina ${ }^{10}(31)$. Al parecer la activación de los receptores es responsabilidad de los tres primeros aminoácidos, en particular de la histidina ${ }^{2}$ y el triptofano ${ }^{3}(31,32)$. La señal termina como consecuencia de tres mecanismos: a) Degradación de la GnRH por proteasas asociadas a la membrana celular; b) Internalización del receptor unido a la GnRH con la subsecuente proteolisis a nivel de los lisosomas y c) reciclaje al exterior con receptores de $\mathrm{GnRH}$ viejos $(32,33)$.

La degradación enzimática de la GnRH ocurre por ruptura de algunos de sus puentes peptídicos. Los estudios in vitro han permitido identificar tres enzimas responsables de la degradación de la hormona: la piroglutamato-aminopeptidasa, la endopeptidasa neutra y la enzima de clivaje post-prolina $(33,34)$. La primera rompe el enlace entre el piroglutamato ${ }^{1}$ y la histidina ${ }^{2}$, la segunda afecta enlaces intermedios, especialmente $\mathrm{Tyr}^{5}$ $\mathrm{Gly}^{6}$, y la última rompe el enlace entre la prolina ${ }^{9}$ y la glicina ${ }^{10}$. Por contraste, los estudios in vivo han identificado como sitios de clivaje los siguientes: Glu $1-\mathrm{Hys}^{2}$, $\mathrm{Hys}^{2}-\operatorname{Trp}^{3}, \operatorname{Trp}^{3}-\mathrm{Ser}^{4}$ y $\operatorname{Ser}^{4}-\mathrm{Tyr}^{5}$ (34).

\section{B. Aumento de la potencia Modificando la molécula de GnRH}

Algunas de las modificaciones de la estructura de la GnRH que dieron lugar a compuestos agonistas mas potentes que la hormona nativa y los posibles mecanismos por los cuales se logra éste aumento de la potencia se resumen a continuación (34):

1. Modificaciones Pro'-etilamida. La substitución de la glicina ${ }^{10}$ por una etilamida produjo un nonapéptido 5 veces mas potente que la GnRH. Esta substitución sugirió que la glicina terminal no era esencial para la potencia de la hormona nativa y que la longitud de la 
cadena jugaba un papel importante en la afinidad del análogo por el receptor.

2. Modificaciones en la glicina ${ }^{6}$. La substitución de la glicina ${ }^{6}$ por D-aminoácidos como la D-Alanina aumentó la potencia del análogo en un 350 a $450 \%$. El efecto se atribuye a que ésta substitución estabiliza la conformación en "V" de la hormona lo cual facilita la interacción con el receptor. Es probable que la substitución de la glicina por D-aminoácidos también vuelva mas resistentes a la degradación enzimática a los enlaces 5-6 y 6-7.

3. Modificaciones con efectos aditivos. La combinación de substituciones de la glicina ${ }^{10}$ por una etilamida y de la glicina ${ }^{6}$ por D-aminoácidos de tipo aromático como el triptófano, produjo efectos aditivos.

4. Modificaciones hidrofóbicas en posición 6. La substitución de la glicina ${ }^{6}$ por aminoácidos hidrofóbicos (como el D-triptófano) hace que la molécula adquiera un carácter mas lipofílico y tenga una vida media mas larga. Este fenómeno puede deberse a un efecto de depósito corporal debido a que el análogo se fija mas fácilmente a las proteínas plasmáticas y al tejido adiposo con lo que disminuye su tasa de depuración renal.

5. Otras modificaciones en el C-terminal. La substitución de la glicina ${ }^{10}$ por el compuesto -aza- Gly ${ }^{10}$ (-NHNHCO-) confiere mayor potencia a la hormona al parecer porque aumenta la resistencia a la degradación por la enzima de clivaje post-prolina que rompe el enlace entre la prolina ${ }^{9}$ y la glicina ${ }^{10}$.

\section{Mecanismo de acción de los análogos de la GnRH}

Los análogos de la GnRH se pueden clasificar en agonistas (también llamados superagonistas) y antagonistas. Los primeros provocan inicialmente una respuesta de liberación de gonadotropinas conocida comúnmente como "flare up", pero con el uso continuo y prolongado ocurre posteriormente el efecto de desensibilización hipofisiaria. A su vez los agonistas pueden ser decapéptidos, si conservan la glicina ${ }^{10}$ o nonapéptidos si la substituyen por una etilamida. Por otra parte, los análogos antagonistas no provocan el efecto de "flare up" sino que, desde la primera administración, producen casi de inmediato una depresión de la síntesis y liberación de gonadotropinas. Al parecer, los antagonistas se unen y ocupan el receptor desplazando a la GnRH natural pero no inducen la dimerización de los receptores, aunque si provocan la internalización de los mismos (35). Mientras el antagonista esté unido al receptor, el gonadotropo no puede reaccionar a la estimulación por la $\mathrm{GnRH}$ natural; por lo tanto, la supresión exitosa de los gonadotropos requiere un suministro constante del antagonista para que los receptores de $\mathrm{GnRH}$ estén ocupados continuamente (32). Los antagonistas también causan un enlentecimiento de los fenómenos de recuperación de la sensibilidad celular. La potencia de un antagonista depende del número de aminoácidos substituidos, así los preparados de tercera generación tienen hasta seis substituciones (31).

Cuando se administra un análogo agonista de la $\mathrm{GnRH}$ (GnRHa), ocurre un aumento inicial de la concentración de FSH y de LH entre el segundo y el tercer día. Si se continúa la administración del análogo, ocurre una disminución progresiva de la LH hasta la línea de base hacia el final de la tercera o cuarta semanas. Por el contrario la concentración de FSH cae a concentraciones por debajo de la línea de base (36). Se ha observado que el descenso de la LH bioactiva es mas pronunciado que el de la $\mathrm{LH}$ inmunodetectable lo que explicaría por qué es posible encontrar concentraciones detectables por inmunoanálisis de $\mathrm{LH}$ en pacientes con tratamientos prolongados con GnRHa (37-39). En parte la disminución de la relación LH inmunodetectable/LH bioactiva en respuesta al uso prolongado de GnRHa se debe a un aumento de la secreción de subunidades libres (36). En el humano el único mecanismo de acción de los GnRHa parece ser la desensibilización a nivel hipofisiario, con la consecuente supresión de las gonadotropinas y en últimas del estradiol $(11,36)$. El estradiol disminuye a valores de la fase folicular temprana y la progesterona se mantiene por debajo de $5 \mathrm{nmol} / \mathrm{L}$

Como sabemos, la actividad fisiológica del gonadotropo depende de que sea estimulado en forma pulsátil por la GnRH (32); la administración de GnRH o de sus agonistas en forma continua produce una disminución importante de la capacidad de respuesta del gonadotropo a través de los mecanismos de regulación descendente (down-regulation) del número de receptores y de desensibilización postreceptor. La regulación descendente del número de receptores ocurre por internalización de los mismos. Ya que los GnRHa tienen una afinidad muy alta por los receptores de la $\mathrm{GnRH}$, son mas resistentes a la degradación enzimática y tienen una farmacocinética diferente, su administración repetida se comporta como una infusión continua de GnRH, causando desensibilización hipofisiaria. La desensibilización posreceptor que producen los GnRHa se debe a: a) Inhibición de la producción de inositol trifosfato mediada por los leucotrienos; b) "Down-regulation" de la proteinkinasa $\mathrm{C}$; c) Disminución de la síntesis de las subunidades de la LH (principalmente de la subunidad $\beta$ por efectos sobre la producción de AMPc y GMPc y d) Inhibición de la glicosilación de la $\mathrm{LH}$ con la consecuente disminucíon de la forma bioactiva de la hormona (8). Las propiedades de los $\mathrm{GnRHa}$ se aprovechan para fines diagnósticos y terapéuticos (Tabla No. 1) $(32,36,40-42)$.

\section{Dosis y administración}

Los GnRHa se pueden administrar por vía intranasal, inyección subcutánea, inyección intramuscular de depósito o en implantes de depósito. La vía intranasal produce alta variación entre los individuos y baja biodisponibilidad del medicamento (1-2\%). En parte éstos efectos se deben a pérdida del análogo por proteolisis y deglución y paso a los senos paranasales. El medicamento puede persistir en la mucosa nasal hasta por 24 horas presentando propiedades similares a las de las formas de depósito (33). No obstante, se requieren inhalaciones frecuentes durante el día (3 a 6) o de lo contrario ocurren fenómenos de escape. Por ésta razón, en la actualidad la vía intranasal se reserva 
Tabla 1

APLICACIONES CLINICAS DE LOS AGONISTAS DE LA GNRH

\section{Alteraciones endocrinológicas}
a. Pubertad precoz
b. Síndrome de ovarios poliquísticos
c. Hirsutismo/acné
d. Diagnóstico de la reserva hipofísiaria
e. Diagnóstico diferencial entre hipogonadismo- hipogonadotropo y la pubertad retardada

\section{Enfermedades ginecológicas e infertilidad}
a. Endometriosis
b. Inducción de ovulación
c. Fertilización in vitro
d. Mastopatía fibroquística severa
e. Hemorragia uterina disfuncional
f. Síndrome de tensión premenstrual

\section{Tratamiento de tumores}
a. Hiperplasia, adenoma y cáncer de la próstata
b. Cáncer del seno
c. Leiomiomatosis

\section{Otros}
a. Contracepción masculina y femenina
b. Reposo gonadal durante la quimioterapia
c. Previo a cirugía histeroscópica
d. Porfiria intermitente aguda

para tratamientos a corto plazo en los que no se requiere una supresión completa, tal es el caso de algunos esquemas de inducción de ovulación (32). Para tratamientos a largo plazo se prefieren en general las otras vías de administración de los GnRHa. La vía subcutánea permite una sola administración diaria del medicamento (32). No obstante, la biodisponibilidad del medicamento cuando se administra por la vía subcutánea puede ser afectada por factores tales como volumen y/o concentración de la inyección, flujo sanguíneo local, trauma de la inyección, densidad de los capilares y/o linfáticos y degradación proteolítica en el sitio de la inyección (33). Cuando se administran por vía intramuscular la tasa de absorción usualmente es mas rápida que cuando se hace por vía subcutánea, por lo que la vía intramuscular se utiliza para las formas de depósito que se administran cada mes. En el caso del acetato de leuprolide de depósito, éste consiste en microesferas liofilizadas del análogo incorporadas en un copolímero biodegradable de ácidos poliláctico y poliglicólico (43). Estas microesferas son degradadas progresivamente por el organismo con lo cual se libera lentamente el análogo. La elevación importante de la concentración del medicamento después de la inyección se debe al leuprolide localizado sobre la superficie de las microesferas pero, después de varias horas, las concentraciones caen a un nivel terapéutico sostenido (43). También existen implantes de liberación lenta y sostenida de algunos GnRHa; así el goserelin se puede administrar mediante la aplicación subcutánea de una pequeña barra de $13 \times 1.2 \mathrm{~mm}$ que contiene $3.6 \mathrm{mg}$ del análogo en un copolímero biodegradable de ácido D-L-lácticoglicoláctico (44). La tolerancia a los preparados intramusculares de liberación lenta es muy buena y por ello constituyen en la actualidad la vía de elección para los tratamientos de larga duración como es el caso de la endometriosis.

Las dosis varían de acuerdo con el tipo de GnRHa que se utiliza y la indicación. Con el fín de minimizar la respuesta agonista inicial o "flare up", el tratamiento de las enfermedades dependientes de estrógenos debe iniciarse en la segunda parte de la fase luteínica del ciclo menstrual, cuando la reserva hipofisiaria de gonadotropinas es menor (31). Sin embargo, éste esquema conlleva el riesgo de iniciar el análogo en un ciclo de embarazo. Para evitar este riesgo la paciente debe utilizar contracepción en el ciclo en el cual se va a iniciar el tratamiento. La duración del tratamiento es variable; para la endometriosis el medicamento se administra usualmente durante un promedio de seis meses; sin embargo, para algunas mujeres un curso de tratamiento de tres meses puede ser suficiente, mientras que para otras la terapia se debe prolongar por nueve a doce meses $(2,45)$.

En la Tabla No. 2 se resumen algunos de los GnRHa y las dosis usuales para el tratamiento de la endometriosis. Como la eficacia terapéutica de los distintos GnRHa es similar y los efectos secundarios son prácticamente los mismos para todos los análogos, la elección del medicamento se hace con base en aquel que resulte mas cómodo para la paciente y que el médico esté mas familiarizado $(14,46)$.

\section{E. Efectos colaterales}

La mayoría de los efectos colaterales resultan de la marcada hipoestrogenemia que se produce durante el tratamiento e incluyen: inestabilidad vasomotora y bochorncs, que ocurren en un $90 \%$ de las pacientes aproximadamente, cefalea, mareo, depresión, ansiedad, fatiga, insomnio y somnolencia, irritabilidad, sudoración, sequedad vaginal, dispareunia, disminución de la líbido, aumento de peso y cambios en los lípidos circulantes (3, $11,14,38,45-49$ ). A pesar de la frecuencia de efectos indeseables, solo un $5 \%$ de pacientes tratadas con $\mathrm{GnRHa}$ 
Tabla 2

DIFERENTES ANALOGOS AGONISTAS DE LA GNRH

\begin{tabular}{|c|c|c|}
\hline COMPUESTO & NOMBRE & DOSIS \\
\hline \multirow[t]{3}{*}{ D-Leu ${ }^{6}$, Pro $^{9}$-NHEt } & Leuprolide & $1.600 \mu \mathrm{g} /$ día VIN \\
\hline & & $3,75 \mathrm{mg} / \mathrm{mes}$ VIM \\
\hline & & $0.5-1 \mathrm{mg} /$ día VSC \\
\hline \multirow[t]{2}{*}{ D-Ser $(t \mathrm{Bu})^{6}$, Pro $^{9}-\mathrm{NHEt}$} & Buserelin & $1.200 \mu \mathrm{g} /$ día VIN \\
\hline & & 200 a $400 \mu \mathrm{g} /$ día VSC \\
\hline D-His $(\mathrm{Bzl})^{6}$, Pro $^{9}-\mathrm{NHEt}$ & Histrelin & $100 \mu \mathrm{g} /$ día VSC \\
\hline $\mathrm{D}-\mathrm{Nal}(2)^{6}-\mathrm{Gly}^{10}$ & Nafarelin & 400 a $800 \mu \mathrm{g} /$ día VIN \\
\hline D-Trp ${ }^{6}-G_{1 y}{ }^{10}$ & Decapeptil & $3,75 \mathrm{mg} / \mathrm{mes}$ VIM \\
\hline $\mathrm{D}-\operatorname{Ser}(\mathrm{t} \mathrm{Bu})^{6}$, aza-Gly ${ }^{10}$ & Goserelin & $\begin{array}{l}3,6 \mathrm{mg} / \mathrm{mes} \text { en } \\
\text { implante subcutáneo }\end{array}$ \\
\hline
\end{tabular}

VIN $=$ vía intranasal $\quad$ VIM $=$ vía intramuscular $\quad$ VSC $=$ vía subcutánea

y un $8 \%$ de pacientes tratadas con danazol suspenden el tratamiento debido a la severidad de los efectos colaterales (13). En un estudio comparativo entre danazol y buserelin se encontró que ambos medicamentos aumentaban significativamente las LDL; sin embargo, mientras que el GnRHa no tuvo efecto sobre las HDL, el danazol las disminuyó significativamente durante todo el tratamiento (3). En ambos casos los cambios en los lípidos circulantes son reversibles al terminar el tratamiento.

Se ha informado que ocurre pérdida de mineral óseo cuando se administran GnRHa para el tratamiento de la endometriosis; por el contrario los datos sugieren que no hay pérdida ósea o incluso hay ganancia ósea en pacientes tratadas con anticonceptivos orales, progestágenos o danazol (13). El tratamiento con GnRHa se relaciona con un aumento del calcio sérico total, el calcio libre, el fósforo, la osteocalcina, la fosfatasa alcalina, las relaciones calcio/creatinina e hidroxiprolina/creatinina urinarias (45), así como alteraciones en los marcadores bioquímicos de remodelación ósea. Estos datos indican que puede haber un aumento de la reabsorción y de la formación ósea durante el tratamiento con GnRHa (45). En dos estudios $(50,51)$ no se encontraron cambios significativos en la densidad ósea media determinada por absorciometría de doble fotón. Sin embargo, en cinco estudios en los cuales se utilizó absorciometría de doble fotón y en otros cinco estudios en los cuales se utilizó tomografía computarizada cuantitativa se encontraron reducciones significativas de la densidad ósea cuando se utilizaron los GnRHa (13). La pérdida de mineral óseo determinada por absorciometría de doble fotón a nivel de la columna vertebral oscila entre un 2 y un $6 \%(43,45,52)$ y la pérdida ósea determinada por tomografía computarizada cuantitativa a nivel vertebral oscila entre el 5 y el $10 \%$ después de seis meses de tratamiento con GnRHa $(45,53)$. Entre mas disminuidas se encuentren las concentraciones de estradiol mayor será la tasa de pérdida ósea. Así, en las mujeres con concentraciones de estradiol de 15 $\mathrm{pg} / \mathrm{mL}$, la pérdida ósea a nivel de la columna vertebral oscila entre el 3 y el 15\% durante los primeros seis meses de hipoestrogenismo (54).

Los cambios de la densidad ósea que se observan con un tratamiento único con $\mathrm{GnRHa}$ son reversibles y no imponen la realización rutinaria de una densitometría ósea en mujeres jóvenes antes del tratamiento; sin embargo, éste procedimiento se debe considerar cuando se va a repetir una terapia con los GnRHa (52). La recuperación de las pérdidas de mineral óseo es, sin embargo, mas lenta de lo que se pensaba anteriormente y pueden persistir déficits aún seis meses a un año después de suspender la terapia (13).

Las concentraciones de estradiol entre 30 y $45 \mathrm{pg} / \mathrm{mL}$ podrían evitar la pérdida ósea sin provocar crecimiento de las lesiones de endometriosis $(2,4)$. De hecho, los regíme- 
nes con GnRHa que solo suprimen el estradiol a $30 \mathrm{pg} / \mathrm{mL}$ mejoran los signos y síntomas de la endometriosis en forma equivalente a los tratamientos en los que el estradiol se suprime hasta $15 \mathrm{pg} / \mathrm{mL}(32,45)$. En consecuencia, las siguientes estrategias permiten lograr supresión de la endometriosis sin deterioro de la densidad ósea $(2,4,13,45)$ : a) utilizar el GnRHa exclusivamente durante tres meses y continuar con el GnRHa con estrógeno + progestágeno en forma continua; b) utilizar un GnRHa durante tres meses y seguir con el GnRHa + un régimen cíclico de estrógeno y progestágeno; c) utilizar un GnRHa a dosis plenas durante tres meses y luego ajustar las dosis del análogo hasta que la concentración de estradiol circulante se encuentre entre 30 y $45 \mathrm{pg} / \mathrm{mL}$; d) utilizar un GnRHa exclusivamente durante tres meses y luego agregarle un progestágeno diariamente; e) utilizar una combinación de GnRHa y danazol y f) utilizar una combinación de GnRHa y calcitonina, bifosfonatos o fluoruros. En un estudio se administraron $3.6 \mathrm{mg}$ de goserelin en inyección de depósito por vía intramuscular cada mes durante seis meses y dos parches semanales de $25 \mathrm{~g}$ de estradiol transdérmico observándose mejoría de la endometriosis, sin que se presentaran efectos adversos; de ésta forma los autores obtuvieron concentraciones plasmáticas de estradiol entre 65 y $250 \mathrm{pmol} / \mathrm{L}(25)$.

Las contraindicaciones de los GnRHa incluyen hipersensibilidad, gestación, hemorragia de etiología no establecida y falla hipofisiaria. También se deben utilizar terapias alternativas en pacientes con historia de osteoporosis $(2,14)$.

\section{F. Resultados del tratamiento de la endometriosis con GnRHa}

La reducción de los puntajes (AFS) de la endometriosis oscila entre el 42 y el $51 \%$ con los diferentes GnRHa y el alivio del dolor varía entre el 69.6 y el $90 \%$ de las pacientes (2). Se ha observado desaparición completa de la endometriosis en un $18 \%$ de pacientes, mejoría importante en un $42 \%$, regresión incompleta en un $12 \%$ y progreso de la endometriosis en un $10 \%$ de las pacientes tratadas con $1200 \mathrm{~g}$ /día de buserelín por vía intranasal. Con nafarelin por vía intranasal se ha informado mejoría hasta en un $87 \%$ de pacientes tratadas para endometriosis (49). El descenso en los puntajes (AFS) ocurre fundamentalmente a expensas de la reducción de los implantes ya que no hay un efecto significativo sobre las adherencias.

En estudios comparativos entre GnRHa y danazol, un $90 \%$ de las pacientes muestran mejoría de los sintomas y la eficacia de los dos medicamentos parece ser similar para inducir la regresión de la enfermedad $(11,43,46,55)$. Tanto los GnRHa como el danazol tardan alrededor de 3 meses para lograr una reducción óptima del dolor pélvico y de la dispareunia, después de los cuales la mejoría se mantiene (13). La disminución en el puntaje (AFS) de la endometriosis oscila alrededor del $50 \%$ tanto para los GnRHa como para el danazol. La regresión de los implantes mayores de $2 \mathrm{~mm}$ es de alrededor del $80 \%$ con cualquiera de los dos tratamientos (46). En cuanto a la fertilidad, no se ha demostrado que los GnRHa aumenten la tasa de embarazos en pacientes con endometriosis cuando se compara con el danazol (15). El grado de supresión del estradiol es mayor en las pacientes tratadas con GnRHa que en las pacientes tratadas con danazol $(11,38,51)$; generalmente las concentraciones de estradiol después de un mes de tratamiento con los GnRHa es menor de $20 \mathrm{pg} / \mathrm{mL}$. No obstante, los GnRHa son mejor tolerados que el danazol (14).

Ocurre una recuperación rápida del ciclo menstrual después de descontinuar la terapia con los GnRHa. Un porcentaje superior al $90 \%$ de las pacientes tratadas durante seis meses tienen su menstruación dentro de las primeras nueve semanas de terminado el tratamiento, algunas presentan menstruación como a las cuatro semanas de suspendido el tratamiento mientras que unas pocas tardan hasta once semanas en recuperar la función menstrual $(38,41)$. Esta rápida reversión de los efectos al suspender los GnRHa puede ser benéfica cuando se trata de pacientes con deseos de fertilidad. Como ocurre con otros tipos de tratamientos conservativos de la endometriosis, puede ocurrir reactivación de los implantes endometriósicos al recuperarse el ciclo menstrual determinando recurrencias de la enfermedad al terminar el tratamiento $(47,49)$. La dismenorrea retorna durante los seis primeros meses; sin embargo, la dispareunia y el dolor pélvico pueden tardar hasta un año en presentarse nuevamente (2).

\section{Tratamiento médico y quirúrgico combinados}

Algunos estudios han informado la presencia de endometriosis microscópica en biopsias peritoneales que visualmente parecían normales $(56,57)$. Si la endometriosis microscópica causa infertilidad, entonces un tratamiento combinado médico y quirúrgico parecería una forma lógica de enfrentar la enfermedad (15). No obstante, en un estudio en el cual se evaluaron las tasas de fertilidad mensual en pacientes tratadas con cirugía únicamente comparadas con pacientes que recibieron danazol antes o después del tratamiento quirúrgico, no se encontraron diferencias significativas entre los tres grupos (15).

Se ha observado que las pacientes con endometriosis severas o extensas pueden beneficiarse con un tratamiento preoperatorio con danazol o GnRHa durante tres meses. Este tratamiento previo reduce la respuesta inflamatoria y la vascularidad, simplificando la cirugía $(2,58)$. El tratamiento médico posoperatorio, por otro lado, suprimiría la enfermedad microscópica en éstos casos (58). Otras pacientes que pueden beneficiarse de un tratamiento posoperatorio con anticonceptivos orales, danazol o GnRHa son las pacientes con severo dolor. Particularmente cuando se trata de mujeres jóvenes que desean preservar la capacidad reproductiva (58).

Resumiendo: aunque el tratamiento quirúrgico extirpa la endometriosis y las secuelas resultantes de la enfermedad, tal terapia no afecta a la enfermedad microscópica 
residual ni a los aspectos sistémicos que acompañan a la endometriosis, tales como los cambios inmunológicos. Por otro lado, la terapia médica no puede corregir las adherencias y la distorsión anatómica que causa la enfermedad y que afectan la fertilidad. Por éstas razones cada vez se utiliza con mas frecuencia la combinación de tratamiento médico y quirúrgico (13).

\section{Conclusión}

El desarrollo de medidas preventivas y de tratamientos que erradiquen completamente la endometriosis se encuentra limitado debido a que se desconoce la etiología e histogénesis de la enfermedad. Los tratamientos hormonales conducen por una u otra vía a una disminución de los esteroides ováricos que soportan el tejido endometriósico. En éste sentido todos los tratamientos hormonales actuarían en forma indirecta sobre la enfermedad. Posiblemente la persistencia de implantes de endometriosis viables que pueden ser estimulados nuevamente por las hormonas ováricas, explica la alta tasa de recurrencias después de suspender la terapia hormonal. En efecto, se ha calculado que la tasa anual de recurrencia después de cualquier tratamiento conservativo de la endometriosis oscila entre un 5 y un $10 \%$ (11); de ésta forma la endometriosis recurre en casi la mitad de los casos dentro de los primeros cinco años después de supendida la terapia. Por lo tanto, hasta el momento los tratamientos conservativos de la endometriosis pueden clasificarse como supresivos pero no curativos de la enfermedad.

Todos los tratamientos endocrinológicos de la endometriosis son eficaces para disminuir la sintomatología y la severidad de la enfermedad; sin embargo, no existe evidencia de que el AMP, el danazol o los GnRHa sean superiores al manejo expectante en cuanto hace referencia al tratamiento de la infertilidad asociada a la endometriosis. Por lo tanto, la elección del tratamiento de la enfermedad debe ser individualizada teniendo en cuenta aspectos médicos tales como la edad de la paciente, el motivo de consulta, la sintomatología, el estado de la enfermedad, el deseo de fertilidad y la presencia o ausencia de compromiso ovárico; aspectos farmacológicos tales como la vía de administración, los efectos colaterales, la tolerancia y las contraindicaciones del medicamento y aspectos sociales tales como los recursos económicos de la paciente. El tratamiento del dolor constituye la indicación primaria de la terapia endocrinológica de la endometriosis; para las pacientes con dolor incapacitante se debe elegir el medicamento que ofrezca alivio lo mas rápido posible. Las pacientes interesadas en fertilidad, especialmente si tienen edad avanzada, deben recibir medicamentos que ofrezcan una recuperación rápida de los ciclos ovulatorios al terminar el tratamiento y se benefician de tratamientos combinados con cirugía y de las técnicas de fertilización asistida. Las pacientes con signos de hiperandrogenismo no deben recibir danazol. También se deben evitar el danazol, los progestágenos y los anticonceptivos orales en pacientes con enfermedades hepáticas. Por otro lado, las pacientes con factores de riesgo para osteoporosis no deben recibir GnRHa. Tampoco se aconseja repetir un tratamiento con GnRHa para pacientes que tengan recurrencias de la endometriosis si han recibido los análogos en los 12 a 18 meses previos; en éstos casos es preferible utilizar un progestágeno o el danazol. En términos de regresión de las lesiones y alivio sintomático, el danazol y los GnRHa ofrecen las tasas mas altas de eficacia, pero a las pacientes con limitaciones económicas se les deben ofrecer otras alternativas.

En la actualidad la práctica mas común consiste en realizar intervenciones terapéuticas durante la videolaparoscopia inicial que se practica para establecer el diagnóstico y, posteriormente, definir la necesidad de un tratamiento médico complementario. Los procedimientos quirúrgicos iniciales se han simplificado y perfeccionado significativamente con el uso de la videolaparocopia e incluyen la aplicación de láser, la electrocauterización, la endocoagulación, la radiocirugía, la lisis de adherencias e incluso la resección de quistes endometriósicos. Las mujeres jóvenes con una enfermedad leve a moderada y deseos de fertilidad se pueden beneficiar con un tratamiento médico o quirúrgico por laparoscopia. En las pacientes con enfermedad severa y/ o con quistes ováricos endometriósicos el tratamiento de elección es el tratamiento quirúrgico. Por otro lado, éste grupo de pacientes con enfermedad severa tiene un pronóstico de fertilidad reservado, y constituye una indicación ampliamente aceptada para la fertilización in vitro cuando hay deseos de embarazo. También se ha informado mejoría de las tasas de embarazo en pacientes con endometriosis manejadas con hiperestimulación ovárica controlada + inseminación artificial y con GIFT.

Teniendo en cuenta la amplia variación biológica que ocurre en la endometriosis y los diferentes mecanismos de acción que tienen los tratamientos endocrinológicos, una buena conducta consiste en utilizar un segundo tratamiento médico diferente, cuando la respuesta al tratamiento inicial no es adecuada.

Eventualmente en el futuro se podrán usar nuevas generaciones de análogos antagonistas de la GnRH evitando el efecto estimulante inicial que tienen los agonistas. La limitante principal que tenían éstos medicamentos era la reacción histamínica severa que provocaban, pero éste efecto se ha venido superando con los antagonistas de tercera generación. A largo plazo el tratamiento de la endometriosis probablemente involucrará moduladores de los factores de crecimiento, sus receptores y sus mediadores posreceptor. Por otro lado, si se demuestra que la endometriosis es una enfermedad sistémica y de origen inmunológico, el tratamiento involucrará en un futuro la utilización de agentes inmunomoduladores. Anticuerpos específicos dirigidos contra moléculas propias del tejido endometriósico parecen ser prometedores para el tratamiento y, acoplados a fluorocromos, podrán servir para la visualización de los implantes peritoneales y su erradicación a través de la laparoscopia (59). 


\section{BIBLIOGRAFIA}

1. Olive DL. Diagnosis and Management of Endometriosis. En: Carr BR, Blackwell RE (Eds.). Textbook of Reproductive Medicine. Appleton \& Lange. Norwalk, Connecticut, USA. 1993; 645.

2. Barbieri RL. Use of GnRH agonists in endometriosis. Infertil Reprod. Med. Clin. North. Am. 1993; 4: 21.

3. Dlugi AM, Rufo S, D'Amico JF, et al. A comparison of the effects of buserelin versus danazol on plasma lipoproteins during treatment of pelvic endometriosis. Fertil Steril 1988; 49: 913.

4. Barbieri RL. Hormonal therapy of endometroisis. Infertil Reprod. Med. Clin. North. Am. 1992; 3: 187.

5. Buttram VC, Reiter RC, Ward S. Treatment of endometriosis with danazol: report of a 6-year prospective study. Fertil Steril 1985; 43 : 353.

6. Buttram VC. Evolution of the revised American Fertility Society Classification of Endometriosis. Fertil Steril 1985; 43: 347.

7. Dmowski WP, Tummon I, Radwanska E, et al. Ovarian suppression in the management of endometriosis: a randomized, comparative study. Fertil Steril 1989; 51: 395.

8. Kiesel L, Runnebaum B. Mechanisms Involved in the Hormonal Treatment of Endometriosis. En: Chadha DR, Buttram VC (Eds.). Current Concepts in Endometriosis. Prog. Clin. Biol. Res. 1990; 323 179.

9. Brosens IA. The rationale for endocrine therapy. Acta Obstet. Gynecol. Scand. (Suppl). 1989; 150: 21.

10. Kauppila AJI, Telimaa S, Rönnber L. Steroidal drugs in endometriosis. Acta Obstet. Gynecol. Scand. (Suppl). 1989; 150: 7.

11. Dmowski P, Rilland R, Schweppe K-W. Endometriosis. Editado por Hoechst. Munich, Alemania. 1989.

12. Moghissi KS. Pseudopregnancy Induced by Estrogen-progestogen or Progestogens Alone in the Treatment of Endometriosis. En: Chadha DR, Buttram VC Jr. (Eds.). Current Concepts in Endometriosis. Prog. Clin. Biol. Res. 1990; 323: 221.

13. Dawood MY. Considerations in selecting appropriate medical therapy for endometriosis. Int. J. Gynecol. Obstet. 1993; 40(Suppl): S29.

14. Hurst BS, Schlaff WD. Treatment options for endometriosis. Medical therapies. Infertil Reprod. Med. Clin. North. Am. 1992; 3: 645.

15. Rosen GF. Treatment of endometriosis-associated infertility. Infertil Reprod. Med. Clin. North. Am. 1992; 3: 721.

16. Luciano AA, Turksoy RN, Carleo J. Evaluation of oral medroxyprogesterone acetate in the treatment of endometriosis. Obstet. Gynecol. 1988; 72: 323.

17. Coutinho EM. Therapeutic Experience with Gestrinone. En: Chadha DR, Buttram VC Jr (Eds.). Current Concepts in Endometriosis. Prog. Clin. Biol. Res. 1990; 323: 233.

18. Thomas EJ, Cooke ID. Impact of gestrinone on the course of asymptomatic endometriosis. Br. Med. J. 1987; 294: 272.

19. Venturini PL, Bertolini S, Brunenghi MCM, et al. Endocrine, metabolic and clinical effects of gestrinone in women with endometriosis. Fertil Steril 1989; 52: 589.

20. Cooke ID, Thomas EJ. The medical treatment of mild endometriosis. Acta Obstet. Gynecol. Scand. (Suppl) 1989; 150: 27.

21. Thomas EJ. Preventive, Symptomatic and Expectant Management of Endometriosis. En: Chadha DR, Buttram VC Jr (Eds.). Current Concepts in Endometriosis. Prog. Clin. Biol. Res. 1990; 323: 197.

22. Donnez J, Nisolle M, Clerckx F, Casanas F. Evaluation of Preoperative Use of Danazol, Gestrinone, Lynestrenol, Buserelin Spray and Buserelin Implant, in the Treatment of Endometriosis Associated Infertility. En: Chadha DR, Buttram VC Jr (Eds.). Current Concepts in Endometriosis. Prog Clin Biol Res 1990; 323: 427 .

23. Mettler L. Pathogenesis, diagnosis and treatment of genital endometriosis. Acta Obstet. Gynecol. Scand. (Suppl) 1989; 150: 31.

24. Barbieri RL. Danazol: Molecular, Endocrine, and Clinical Pharmacology. En: Chadha DR, Buttram VC Jr (Eds.). Current Concepts in Endometriosis. Prog. Clin. Biol. Res. 1990; 323: 241

25. Maouris P, Dowsett $M$, Rose $G$, et al. A new treatment for endometriosis. The Lancet 1989: 1018.

26. Trabant $\mathrm{H}$, Widdra W, de Looze S. Efficacy and Safety of Intranasal Buserelin Acetate in the Treatment of Endometriosis: a Review of Six Clinical Trials and Comparison with Danazol. En: Chadha DR,
Buttram VC Jr (Eds.). Current Concepts in Endometriosis. Prog Clin Biol Res 1990; 323: 357.

27. Chong A, Keene ME, Thornton NL. Comparison of three modes of treatment for infertility patients with minimal pelvic endometriosis. Fertil Steril 1990; 53: 407.

28. Rosenfield RL, Jacob J. Subsequent pregnancies in previously infertile women with endometriosis. Obstet. Gynecol. 1988; 72: 908.

29. El-Roeiy A, Dmowski WP, Gleicher N, et al. Danazol but not gonadotropin-releasing hormone agonists suppresses autoantibodies in endometriosis. Fertil Steril 1988; 50: 864.

30. Gleicher N, Pratt D. Abnormal (auto)immunity and endometriosis. Int J Gynecol. Obstet. 1993, 40(Suppl): S21.

31. Calaf J. Análogos de la GnRH: pasado, presente y futuro. Drugs of Today 1990; 26(Supl. 5): 1.

32. Henzl MR. Hormona liberadora de gonadotropinas y sus análogos: del laboratorio a la cabecera. Clin. Obstet. Ginecol. 1993; 3: 588.

33. Handelsman DJ, Swerdloff RS. Pharmacokinetics of gonadotropinreleasing hormone and its analogs. Endocrine Rev 1986; 7: 95.

34. Karten M, Rivier JE. Gonadotropin-releasing hormone analog design. Structure-function studies toward the development of agonists and antagonists: rationale and perspective. Endocrine Rev 1986; 7: 44.

35. Hazum E, Conn M. Molecular mechanism of gonadotropin releasing hormone (GnRH) action. I. The GnRH receptor. Endocrine Rev 1988; 9: 379 .

36. Bhasin S, Swerdloff RS. Mechanisms of gonadotropin-releasing hormone agonist action in the human male. Endocrine Rev 1986; 7 : 106.

37. Jaakkola T, Ding Y-Q, Kellokumpu-Lehtinen P. The ratios of serum bioactive/immunoactive luteinizing hormone and follicle-stimulating hormone in various clinical conditions with increased and decreased gonadotropin secretion: reevaluation by a highly sensitive immunometric assay. J Clin Endocrinol Metab 1990; 70: 1496.

38. Steingold KA, Cedars M, LH JKH, et al. Treatment of endometriosis with a long acting gonadotropin-releasing hormone agonist. Obstet. Gynecol. 1987; 69: 403.

39. Lemay A, Bergeron J, Sandow J, et al. Escape from the downregulation of the pituitary-ovarian axis following decreased infusion of luteinizing hormone-releasing hormone agonist. Fertil Steril 1988; 49: 802 .

40. Corbin A, Bex FJ, Jones RC. Comparison of LHRH agonist and antagonist: antifertility and therapeutic developments. En: Labrie F, Belanger A, Dupont A (Eds.). LHRH and its analogues. Elsevier Science Publishers BV. 1984; 95.

41. Lemay A, Maheux R, Faure N, et al. Reversible hypogonadism induced by a luteinizing hormone-releasing hormone (LH-RH) agonist (Buserelin) as a new therapeutic approach for endometriosis. Fertil Steril 1984; 41: 863.

42. Neyro JL. Miscelánea: indicaciones, alternativas y otros usos de los análogos LH-RH. Drugs of Today 1990; 26(Supl. 5): 37.

43. Miller JD. Leuprolide acetate for the treatment of endometriosis. En: Chadha DR, Buttram VC Jr (Eds.). Current Concepts in Endometriosis. Prog. Clin. Biol. Res. 1990; 323: 337.

44. Shaw RW. Goserelin-Depot preparation of LHRH analogue used in the treatment of endometriosis. En Chadha DR, Buttram VC Jr (Eds.). Current Concepts in Endometriosis. Prog. Clin. Biol. Res. 1990; 323: 383.

45. Barbieri RL. Agonistas de la hormona liberadora de gonadotropinas: tratamiento de la endometriosis. Clin. Obstet. Ginecol. 1993; 3: 606

46. Ordáz J, Pérez-Piñar. Análogos de LH-RH en el tratamiento de la endometriosis. Drugs of Today 1990; 26(Supl. 5): 11.

47. Zorn J-R, Risquez F, Comaru-Schally, Schally AV. Treatment of endometriosis with a delayed release preparation of the agonist Dtrp 6 -luteinizing hormone-releasing hormone: long-term follow-up in a series of 50 patients. Fertil Steril 1990; 53: 401 .

48. Weise HC, Fiedler K, Kato K. Buserelin suppresion of endogenous gonadotropin secretion in infertile women with ovarian feedback disorders given human menopausal/human chorionic gonadotropin treatment. Fertil Steril 1988; 49:399.

49. Scriock E, Monroe SE, Henzl M, et al. Treatment of endometriosis with a potent agonist of gonadotropin-releasing hormone (nafarelin)". Fertil Steril 1985; 44: 583 
50. Damewood M, Schlaff WD, Hesla JS, Rock JA. Interval bone mineral density with long term gonadotropin-releasing hormone agonist suppression. Fertil Steril 1989; 52: 596.

51. Tummon IS, Radwanska E, Ali A, et al. Bone mineral density in women with endometriosis before and during ovarian suppresion with gonadotropin-releasing hormone agonists or danazol. Fertil Steril 1988; 49: 792.

52. Henzl MR, Monroe SE. Nafarelin: a new medical therapy for endometriosis. En: Chadha DR, Buttram VC Jr (Eds.). Current Concepts in Endometriosis. Prog. Clin. Biol. Res. 1990; 323: 343.

53. Fogelman I. Gonadotropin-releasing hormone agonists and the skeleton. Fertil Steril 1992; 57: 715.

54. Dawood MY, Lewis V, Ramos J. Cortical and trabecular bone mineral content in women with endometriosis: effect of gohadotropin releasing hormone agonist and danazol. Fertil Steril 1989; 52: 21.
55. Henzl MR, Corson SL, Moghissi K, et al. Administration of nasal nafarelin as compared with oral danazol for endometriosis: A multicenter double-blind comparative trial. N. Engl. J. Med. 1988; 318: 485 .

56. Nisolle M, Paindaveine B, Bourdon A, et al. Histologic study of peritoneal endometriosis in infertile women. Fertil Steril 1990; 53: 984.

57. Murphy AA, Green WR, Bobbie D, et al. Unsuspected endometriosis documented by scanning electron microscopy in visually normal peritoneum. Fertil Steril 1986; 46: 522.

58. Malinak LR. Surgical treatment and adjunct therapy of endometriosis. Int. J. Gynecol. Obstet. 1993; 40(Suppl): S43.

59. Simón C. Endometriosis. El futuro. En: Simón C, Nezhat C. (Ed). Cuadernos de Medicina Reproductiva. Volumen 1, No. 2. Endometriosis. Editorial Panamericana, Madrid, España. 1995. 